\title{
Leur remonter les bretelles ou leur faire passer des ceintures?
}

\author{
Jean-Michel Génevaux, Samuel Gougeon, Jean-Pierre Dalmont, Nicolas Joly, A. Pelat \\ jmgenev@univ-lemans.fr \\ ENSIM, rue aristote, 72000 Le Mans
}

RESUME : Au sein de l'Université du Maine, pour plusieurs modules, une méthode d'évaluation par passage de ceintures est proposée aux étudiants en lieu et place d'un examen terminal. S'inscrivant dans une démarche d'évaluation à la fois certifiante et formative, respectant le rythme d'acquisition de chacun, les modalités de création et de fonctionnement des ceintures montrent que la validation progressive par ceintures est sécurisante. Les auteurs, tous enseignants, donnent leur réactions et points de vues, et interprètent les réactions et les points de vues de l'institution et des étudiants. Un bilan chiffré sur deux années des choix et stratégies des étudiants sont présentés.

Mots clés : dispositif d'évaluation, évaluation formative, ceintures, Freinet, Oury

\section{1 ÉVALUATION : DES OBJECTIFS, DES MODALITÉS ET DES PARTENAIRES MULTIPLES}

L'évaluation comporte usuellement trois phases : a) l'évaluation initiale, ou diagnostic; b) l'évaluation formative, ou entraînement ; c) et l'évaluation finale, souvent sommative et attendue comme certifiante. Les modalités d'évaluation - auto-évaluation ou par les pairs ou par le formateur ou par un tiers ; progressive au fil des enseignements, ou seulement finale; surveillée, ou autonome... -- dépendent étroitement des objectifs, des moyens, et des enjeux de celle-ci.

Le diagnostic initial devrait s'imposer chaque fois que le groupe attendu est hétérogène en niveaux, et qu'une pédagogie individualisée conséquente est possible et envisagée, au moins durant une phase initiale de mise à niveau. Outre la diversité des cursus antérieurs observable au sein d'une promotion, on peut constater, lors du recrutement des étudiants, et malgré -- en France et en Europe -- le découpage des compétences en modules marqués et crédités, que les compensations opérées entre modules en amont dans les cursus ne sont pas rares et brouillent la lisibilité des compétences réellement acquises. Sans ce brouillage, cette certification antérieure suffirait comme diagnostic pour la suite (module suivant, ou année suivante).

L'évaluation formative s'applique aux apprentissages en cours et consiste le plus souvent en exercices à traiter de manière autonome, accompagnés des réponses (mais pas nécessairement des solutions intégrales) -- éventuellement différées --, dont la consultation par l'étudiant constitue une validation. La diffusion d'annales corrigées contribue à ce type d'évaluation. La question de l'individualisation du rythme de l'apprentissage est ici posée. Elle est fortement couplée et contrainte par les modalités retenues pour la certification des compétences acquises.

Une certification classique est le plus souvent organisée sous forme d'une séance d'examen durant laquelle le même sujet abordant un ensemble parfois large de notions est distribué à tous les étudiants du groupe au même moment. Les raisons de ces contraintes très fortes sont connues et tout aussi fortes : elles relèvent essentiellement de la nécessité de maîtriser rigoureusement les conditions pratiques de la certification, avec des moyens (en temps.homme) raisonnablement limités. Bien que légitime, cette économie a des coûts cachés que la dégradation du niveau moyen de compétences de base d'une classe d'âge d'étudiants révèle ou ne peut plus masquer [1].

Trois partenaires sont demandeurs d'évaluation. Tout d'abord, l'évaluation des étudiants est un acte que l'institution impose à chaque enseignant, car elle doit garantir que le titre qu'elle délivre est fondé sur les acquisitions de connaissances et/ou de compétences telles que décrites dans le syllabus qui a permis l'habilitation de la formation. L'évaluation des étudiants est aussi pour l'enseignant une mesure de l'efficacité de la transmission des concepts, des idées, des méthodes, envers le public qu'il forme. Mais l'évaluation est aussi pour l'étudiant l'un des moyens de savoir qu'il sait, et donc d'observer sa propre croissance et le rassurer vis-à-vis des responsabilités qu'il aura par la suite, qu'elles soient professionnelles ou personnelles.

La délivrance du diplôme, faite par un jury de fin d'année ou de semestre, nécessite que le bilan des compétences acquises soit à sa disposition le jour de sa tenue. Il n'y a pas donc de lien formel entre la date de fin d'un module de formation et cette date de bilan si ce n'est l'antériorité.

Proposition est faite dans cet article, de confronter les modalités de l'examen final de fin de module, au souhait des trois partenaires de l'évaluation, et les temporalités différentes de l'acte d'évaluer et du jury.

Inspirés des ceintures mises en place en pédagogie institutionnelle dans des classes de primaire ou de collège [2][3], plusieurs enseignants de l'Université du 
Maine ont proposé aux étudiants d'être évalués par ceintures.

\section{MIEUX RESPECTER LES DIFFERENCES DE RYTHME, POUR MIEUX ASSIMILER}

Un constat pourra probablement faire consensus : même lorsqu'ils partent avec des niveaux de maîtrise d'une compétence pré-requise proches, les étudiants apprennent différemment, à différentes vitesses, avec différentes sensibilités, sous différentes contraintes: certains doivent être salariés en parallèle de leur études, d'autres non; certains travaillent plus facilement avec des livres et documents écrits, d'autres ont plus besoin d'interagir avec les formateurs; certains approfondissent plus mais sont plus lents ; etc.

L'organisation classique de séances de travaux dirigés durant lesquelles tous les étudiants traitent du même sujet au même rythme avec une correction délivrée au tableau pour tous au même moment suppose implicitement que les différences de rythmes sont gommées par des différences d'investissement personnel consenti au fil des séance, mais hors séances. En pratique, nous savons que cette hypothèse est en tout ou partie illusoire. Une organisation alternative -- essentiellement asynchrone -- du travail personnel en TD et de l'évaluation formative afférente peut être envisagée : les étudiants d'un groupe suivent un programme et une démarche communs, mais chacun à son rythme. Ce type d'organisation est mis en œuvre par quelques enseignants à l'ENSIM depuis 2011 [4].

Une réflexion concernant les modalités de la certification découle naturellement de l'organisation des TD modifiée en amont. Imposer à tous une date unique d'examen final revient soit à interrompre l'apprentissage des plus lents, soit à faire attendre (parfois longtemps) les plus rapides. Or un des rôles de la certification consiste à assoir la confiance qu'un étudiant peut avoir en lui-même, en asseyant la confiance que les tiers peuvent avoir en ses compétences, l'aidant ainsi à envisager la suite de manière cohérente et conséquente. La certification est une reconnaissance. Elle donne du sens à son travail et à son cursus.

\section{CERTIFICATION}

Tout d'abord, les visions des trois partenaires à propos de l'examen final sont présentées. Dans un second temps, les modalités de fonctionnement par ceintures sont explicitées avant que les trois partenaires ne donnent leur ressentis. Quelques chiffres, présentés au $\S 4$, essayent alors d'éclairer l'évolution sur deux années de ce système d'évaluation par ceintures à l'Université du Maine

\subsection{Certification classique : l'examen terminal}

\subsubsection{Pour l'institution}

La simplicité temporelle de l'examen terminal, une date, un créneau horaire et une salle, fait que ce mode d'évaluation est très aisé à mettre en place, en prévoyant un temps suffisant entre examen et jury pour la correction des copies.

\subsubsection{Pour l'étudiant}

Imposer une date d'évaluation unique expose les étudiants à deux risques, souvent appréhendés : pour les plus lents, d'être testés sans avoir le sentiment d'être prêts, si la certification ne les attend pas; pour les plus rapides, de devoir se "remettre dans le bain" ou de risquer d'avoir partiellement oublié, si la certification attend les plus lents.

Un bachotage acharné la veille au soir de l'examen, en espérant décrocher la moyenne le lendemain, semble être une attitude classique de certains étudiants. L'objectif peut devenir alors de " sauver les meubles », et de «tourner la page» pour ce consacrer ensuite à une autre matière.

De plus, compte tenu du coté plutôt aléatoire des examens, un étudiant très médiocre peut parfois réussir, et un étudiant dans la moyenne échouer sur un sujet particulier pour une incompréhension de début.

Il ne reste plus qu'à l'enseignant voyant venir la catastrophe avant l'examen : « Il faudrait au moins, que vous refassiez les exercices que nous avons fait ensemble en td !", à remonter les bretelles aux étudiants ou à passer à un mode d'évaluation par ceintures.

\subsubsection{Pour l'enseignant}

L'examen terminal a pour intérêt pour l'enseignant de n'avoir qu'une seule copie par étudiant à corriger. Par contre, la progressivité de l'examen peut empêcher l'enseignant, si l'étudiant échoue dans la première partie du devoir, de se forger une opinion sur sa compréhension des concepts et méthodes utiles au reste du devoir. Son exigence se doit d'être abaissée, en mettant des points sur la méthode de résolution, même si les résultats chiffrés sont faux, voir absurdes. Il peut encore espérer un aspect formateur de cette évaluation pour les quelques étudiants qui daigneront consulter leur copie afin de comprendre leurs erreurs (à moins que ce ne soit pour revendiquer une augmentation de la note), la majorité se contentant de la connaissance de leur note. 


\subsection{Certification alternative : les ceintures}

\subsubsection{Les modalités de création et de fonctionnement de ceintures}

Pour un module, l'enseignant construit un arbre d'enchaînement de ceintures, les flèches indiquant l'ordre de passage de celles-ci, chacune testant une étape de la formation et contribuent, sous forme de points, au calcul de la note finale (Fig. 1). Il publie les compétences testées et le nombre de points acquis pour chacune des ceintures, le nom du correcteur, la date maximale de passage et le nombre maximal de tentatives s'il existe. Des exemples de découpages en ceintures sont accessibles dans les polycopiés disponibles sur des sites d'enseignement [5] [6] [7] [8] [9].

L'enseignant propose individuellement aux étudiants d'être évalués soit par ceintures, soit par un examen terminal classique. Un délai d'une à deux semaines est laissé à chacun pour exprimer son choix. L'enseignant garde la maîtrise du mode d'évaluation, et peut refuser l'évaluation par ceintures à certains étudiants, par exemple en l'encontre de ceux ayant rompu leur engagement sur l'honneur pour des ceintures passées dans d'autres matières.

Un étudiant qui a choisi une évaluation par ceintures, lorsqu'il souhaite s'évaluer pour une ceinture particulière, s'inscrit à une session via un site web [10]. A l'université du Maine, depuis la rentrée 2012, ces sessions ont lieu une fois par semaine et sont mutualisées pour toute l'université. Les passages de ceintures s'arrêtent 10 jours avant le jury de fin de semestre. En entrant dans la salle l'étudiant signe sur sa copie l'engagement : "Je m'engage sur l'honneur à n'évoquer avec personne le contenu du sujet de passage de cette ceinture. Cependant, dans le cas où je ne réussirais pas à l'obtenir, j'ai compris pouvoir discuter de mon travail avec les étudiants ayant acquis cette ceinture. Si l'enseignant à l'intime conviction que je n'ai pas respecté mon engagement, je ne pourrai plus passer de ceintures dans la matière concernée pour l'année universitaire en cours, et je déclare accepter de n'avoir aucun recours vis-à-vis de sa décision." Il reçoit le sujet, soit celui correspondant à la couleur et au numéro de sa tentative, soit celui du jour pour une couleur (tous les étudiants ayant alors le même), il compose et rend copie, sujet et brouillons.

L'enseignant corrige les copies, et informe de l'obtention ou non de la ceinture. Il est à disposition des étudiants pour leur expliquer les raisons d'un éventuel refus d'attribution.

En cas d'échec, l'étudiant peut retenter la ceinture sur un autre sujet. Dans le cas où le nombre de tentatives d'une même ceinture n'est pas limité, à partir de la $6^{\text {ième }}$ tentative, un sujet est tiré au sort parmi les 5 versions existantes.

La figure 1 présente un exemple d'arbre de progression de ceintures au sein d'une unité d'enseignement. Pour le thème "dynamique des structures», les ceintures jaune et orange sont en parallèle car aucune n'a besoin de prérequis évalués dans l'autre. Par contre le passage de la ceinture verte nécessite l'obtention des deux ceintures jaune et orange. La contribution de chaque ceinture à la formation de la note est définie par un certain nombre de points à chaque ceinture. Si un barème propose un total supérieur à 20 points cela autorise de fait la compensation.

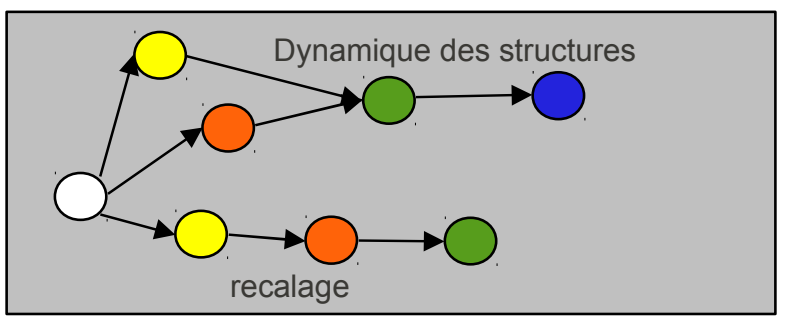

Fig. 1 : Arbre d'enchaînement des ceintures en dynamique des structures et recalage pour l'année 2012-2013.

\subsubsection{Positionnements et réactions de l'institution}

L'institution est naturellement rétive à l'apparition de perturbations qui lui apparaît remettre en cause ses fonctionnements.

Par exemple à l'Université du Maine, l'année 20112012 où des ceintures ont été introduites, les modalités de contrôle des connaissances qui ont été votées en CA de l'ENSIM affichaient pudiquement comme mode d'évaluation du module concerné la modalité «évaluation» au lieu de la modalité «ceintures ou examen » telle que demandée par l'équipe enseignante. Même si l'institution soutenait l'expérimentation, elle hésitait à afficher ce soutien. L'année suivante, après une année de fonctionnement par ceintures pour plusieurs modules, les modalités de contrôle des connaissances étaient votées avec une mention ouvertement affichée « ceintures ou examen ».

Cette année 2012-2013, le nombre de modules évalués par ceintures à l'Université du Maine est croissant et ceci dans plusieurs composantes de l'université. Il a donc été proposé de présenter cette modalité au Conseil des Études et de la Vie Universitaire. Pour des « raisons d'agenda » ceci a été reporté.

Ce type de réactions de la structure est l'une de celle décrite par Imbert [11]. 


\subsubsection{Positionnements et réactions de l'étudiant}

Avec les ceintures, le coté aléatoire de l'examen s'estompe : les compétences demandées pour réussir une ceinture sont clairement spécifiées au départ et circonscrites. L'absence ou l'invisibilité des liens entre les exercices d'un sujet d'examen sommatif terminal fait place à une évaluation mettant en exergue une construction progressive, cohérente, et sécurisée par étapes. Ce changement d'approche responsabilise l'étudiant, alors que tout élément aléatoire tend a contrario à invoquer la fatalité plus que la responsabilité. Un sujet sommatif cohérent mène aux problèmes plus longs, mais le risque de blocage sur les premières questions devient alors non négligeable.

L'étudiant comprend rapidement le dilemme : il faudra travailler pour réussir mais d'un autre coté s'il travaille un minimum il a l'assurance d'avoir la moyenne. A contrario, s'il ne s'entraine pas il est aussi presque certain d'échouer. Il est le seul acteur pour résoudre ce cruel dilemme.

Échouer à une ceinture n'a rien de grave, car l'étudiant vient généralement consulter sa copie pour comprendre son erreur, ce qui lui permettra à la tentative suivante de montrer qu'il ne la fait plus. Des échecs successifs à une même ceinture, déclenche logiquement la consultation. L'évaluation devient formative de façon incontournable pour lui.

De plus, le positionnement de chacun dans cet arbre de ceintures par rapport à l'ensemble des ceintures obtenues par le groupe constitue un signal qui peut être décisif, autant pour l'étudiant que pour le formateur. Ce positionnement affiché permet à l'étudiant détenant une ceinture, de devenir personne ressource pour les autres.

L'obtention progressive de ceintures joue le rôle de cliquet: l'étudiant ne peut que se voir progresser, ce qui est naturellement valorisant.

\subsubsection{Positionnements et réactions de l'enseignant}

Selon l'enseignant, ce système des ceintures est l'occasion de mettre fin à l'examen "loterie" dans lequel l'étudiant vient en ayant peu ou pas travaillé mais en se disant qu'avec un peu de chance il pourra faire quelque chose. Une telle attitude est fréquente à l'université lorsqu'un système de compensation permet la validation d'un module malgré un certain nombre de mauvaises notes.

Il est tout à fait intéressant pour l'enseignant d'observer la réaction des étudiants face à la mise en place ce nouveau système lorsqu'il fait suite à un examen traditionnel. Certains étudiants sont venus, en se disant qu'avec un peu de chance... et ont constaté que l'examen était hors de portée pour eux. Ils se sont alors présentés à la première ceinture sans avoir plus travaillé et ont donc échoué. C'est seulement à ce moment là qu'ils ont compris que cela demandait un minimum d'investissement ! Lors de la seconde tentative on constate que l'ensemble des étudiants avait travaillé, parfois collectivement, pour réussir cette satanée ceinture: nous avions enfin pu mettre les étudiants au travail ! C'est selon nous, la principale vertu de ce système, l'objectif étant à terme que l'étudiant comprenne qu'en travaillant il progresse et, rêvons, qu'un jour il prenne goût au travail !

Pour l'enseignant, un taux d'échec trop grand au passage de la première tentative d'une ceinture, est un bon indicateur d'une « marche » trop haute par rapport à la ceinture précédente, ou qu'il lui faut insister sur un point particulier dans son enseignement et proposer un exercice qui permette de vérifier l'acquisition par l'étudiant d'un point spécifique qui l'a fait échoué, avant qu'il ne se représente à la ceinture. Une réactivité plus grande de l'enseignement est mise en place. La méthode mène à une meilleure connaissance des aptitudes et difficultés de chaque étudiant. Elle autorise à rétro-agir de manière adaptée et individualisée en séances de TD.

Le nombre de corrections à effectuer est bien plus important pour l'enseignant et la gestion des sujets et copies plus complexe. Si la méthode de validation en « tout -ou-rien» est choisie (aucune erreur dans la copie), la correction de chaque copie peut-être extrêmement rapide, et permet d'envoyer des signaux forts concernant les compétences clés, sans pour autant hypothéquer l'avenir. Une disponibilité de l'enseignant sur un créneau horaire de la semaine est inévitable pour que les étudiants puissent savoir pourquoi une ceinture leur a été refusée. Le taux de consultation de copies est bien plus grand que suite à un examen terminal.

Si un étudiant a validé les ceintures de plus bas niveau, il est alors possible à l'enseignant de tester la compréhension d'éléments avancés d'un cours.

La gestion de la salle de passage de ceintures est assez complexe : multiplicité des sujets, départs échelonnés, vérification de non sortie de sujets et de feuilles pour éviter les fuites. La valeur de la parole donnée peut ne pas être la même au sein d'une classe de primaire et dans un groupe de 179 étudiants venant de formations différentes.

\section{QUELQUES ÉLÉMENTS CHIFFRÉS}

\subsection{Le choix des étudiants}

Pendant l'année 2011-2012, les ceintures ont été proposées à plusieurs groupes. Les 35 étudiants de 3ième année d'école d'ingénieur ont tous choisi d'être 
évalués par ceintures. Les 60 étudiants de $2^{\text {nd }}$ année d'école d'ingénieur ont tous choisi d'être évalués par ceintures, dont 2 ont été arrêtés dans leurs passages de ceintures pour non respect de l'engagement qu'ils avaient signé.

Pendant l'année 2012-2013, les choix des étudiants sont résumés dans le tableau 1. Les étudiants en 3ième année d'Ensim, qui ont déjà testé l'évaluation par ceintures lors de leur seconde année ont tous renouvelés ce choix. Parfois les taux peuvent varier en cours d'année, si dans un module, les étudiants sont autorisés à basculer de la modalité examen à la modalité ceintures lorsqu'ils découvrent le sujet de l'examen en entrant dans la salle pour composer : ce fut le cas des 15 étudiants M1 mécanique qui ont alors choisi les ceintures.

Tab.1 : Nombre d'étudiants en fonction de leur choix d'une modalité de contrôle en 2012-2013

\begin{tabular}{|c|c|c|}
\hline formation & examen & ceintures \\
\hline Ensim 1A & 3 & 63 \\
\hline Ensim 2A & 3 & 42 \\
\hline Ensim 3A & 0 & 22 \\
\hline LPAV & 8 & 12 \\
\hline M1 acoustique & 0 & 17 \\
\hline M1 mécanique & $15(0)$ & $50(65)$ \\
\hline
\end{tabular}

\subsection{Les connaissances acquises}

La figure 2 présente l'histogramme de répartition des ceintures maximales atteintes par les étudiants de seconde année d'école d'ingénieur dans 3 modules en 2011-2012.

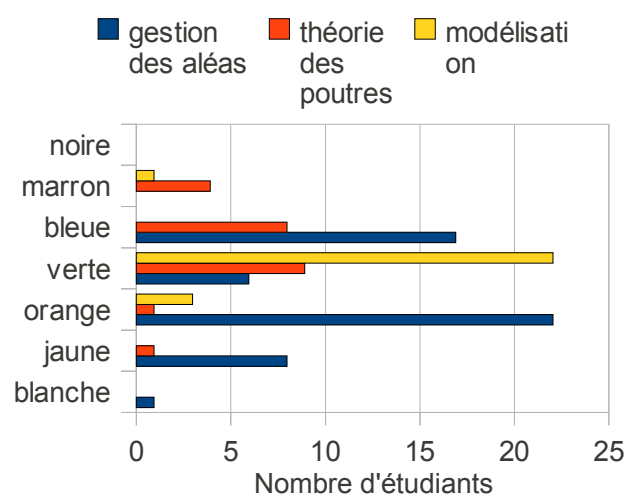

Fig. 2 : Histogramme des ceintures maximales obtenues par les étudiants dans trois disciplines au cours de l'année 20112012.

On constate que une partie des étudiants s'arrêtent de passer les ceintures une fois la moyenne au module obtenue. Parfois, tout en fin de semestre, réapparaissent des étudiants, qui souhaitent gagner quelque points dans ce module, pour compenser des déficits dans d'autres matières qui font parties de la même Unité d'Enseignement.

L'enseignant, peut de plus garantir, pour chaque étudiant et dans le détail, les compétences qu'il a validées. Le nombre de tentatives utilisées pour obtenir une ceinture est délibérément ignoré : l'important est que la compétence soit validée en fin de semestre.

\section{CONCLUSION}

La mise en place d'une évaluation par ceintures permet à chaque étudiant d'être maître du rythme de ses évaluations. L'augmentation du nombre d'étudiants utilisant ce mode d'évaluation à l'Université du Maine ne va pas sans poser des problèmes techniques de gestion de la salle de passage de ceinture, ce qui oblige l'équipe enseignante à en optimiser rapidement l'organisation par une procédure cadrée.

Redonner aussi la main à l'étudiant sur son rythme d'apprentissage, peut être un complément intéressant que certains enseignants utilisent [3].

On pourrait regretter que ce système arrive beaucoup trop tard (en M1 parfois). C'est dès la L1 que ce système devrait être mis en œuvre car pour l'instant le gâchis est énorme : nombre d'étudiants arrivent sans avoir assimilé des compétences qui relèvent du programme du lycée, voir du collège.

Par cet article, nous témoignons d'une démarche contribuant à y remédier. Le coût en temps afférent en est élevé, et nous travaillons à améliorer et alléger la démarche sans en perdre les vertus. Il n'y a cependant pas de mystères : tenir réellement compte de la baisse du niveau d'entrée des étudiants, sans transiger sur leur niveau de compétences professionnelles en sortie de cursus ne s'improvise pas: de nouvelles méthodes pédagogiques doivent être explorées.

\section{Bibliographie}

[1] E. Amara, J.-P. Amar, «Education nationale : un grand corps malade » (Canal+ / Bonne compagnie), 2007 : http://www.youtube.com/watch? $v=p G K G y F m H q I s$

[2] F Oury A. Vasquez "De la classe coopérative à la pédagogie institutionnelle", Ed. François Maspero, Paris, 1981

[3] S. Connac, ""Apprendre avec les pédagogies coopératives", ESF éditeur, Thiron

[4] J.M. Génevaux, A. Pelat, "Collaborative learning in an University", actes du colloque Acoustic's 2012, 23-27 April 2012, Nantes, France, paper 230. hal

[5] J.-M. Génevaux :

ENSIM 1A "Éléments de remise à niveau : Physique", http://umtice.univ-lemans.fr/mod/resource/view.php? $\mathrm{id}=10094$

ENSIM 2A "Statique des poutres" http://umtice.univ-lemans.fr/mod/resource/view.php? id $=10299$ 
ENSIM 2A "Gestion des aléas"

http://umtice.univ-lemans.fr/mod/resource/view.php?

id $=10119$

ENSIM 2A "Modélisation"

http://umtice.univ-lemans.fr/mod/resource/view.php?

$\mathrm{id}=10330$

ENSIM 3A "Dynamique des structures",

http://umtice.univ-lemans.fr/mod/resource/view.php?

id $=10333$

LPAV "Bases de l'acoustique"

http://umtice.univ-lemans.fr/mod/resource/view.php? id=29099

[6] S. Gougeon ENSIM 1A "Éléments de remise à niveau : Thermique, Ondes",

[7] J.-P. Dalmont Master1 "Acoustique",

[8] A. Pelat ENSIM 2A "Acoustique",

[9] N. Joly ENSIM 1A "Éléments de remise à niveau : Physique",

[10] J.-M. Génevaux et al. "Ceintures à l'Université du Maine"

http://umtice.univ-lemans.fr/course/view.php?id=262

[11] F. Imbert , "Innovation et temporalité" , Revue française de pédagogie. Volume 75, 1986. pp. 53-59 